\title{
AN EXTENSION OF THE LEHMERS' PICTURESQUE EXPONENTIAL SUMS
}

\author{
TOM M. APOSTOL
}

Dedicated to to the memory of D. H. Lehmer

\begin{abstract}
A class of exponential sums studied by D. H. Lehmer and Emma Lehmer is generalized by replacing a root of unity by an arbitrary complex number $x$.
\end{abstract}

\section{INTRODUCTION}

Throughout their long and illustrious careers as number theorists, D. H. Lehmer and Emma Lehmer, both individually and in collaboration, used computers to enhance their research. In two joint papers on "Picturesque Exponential Sums" [1, 2] they obtained striking geometric diagrams by plotting the values of certain exponential sums in the complex plane as vertices of graphs connected by line segments. Specifically, they considered the sums

$$
S_{j}(m)=\sum_{n=0}^{m} \zeta^{b(n)+j n},
$$

where $\zeta=\zeta_{k}=\exp (2 \pi i / k)$ and $b(n)$ is the sum of the digits of $n$ when written to the base $k$, where $k$ is a fixed integer $\geq 2$. In other words, when $n$ is written in the form

$$
n=d_{0}+d_{1} k+d_{2} k^{2}+\cdots \quad\left(0 \leq d_{i}<k\right),
$$

we have

$$
b(n)=d_{0}+d_{1}+d_{2}+\cdots .
$$

The principal theorem of paper [1], which they called The Rotation Theorem, states that

$$
S_{j}\left(n k^{2}+d_{1} k+d_{0}\right)=\zeta^{b(n)} S_{j}\left(d_{1} k+d_{0}\right) .
$$

This shows that the sum $S_{j}(m)$, although not a periodic function of $m$, cannot take on more than $k^{3}$ values. In fact, all the different values of $S_{j}(m)$ are found among its first $k^{3}$ values. The picturesque nature of the Lehmer sums depends in large part on the rotation property.

This note generalizes $S_{j}(m)$ by replacing the factor $\zeta^{j}$ in the summand with an arbitrary complex number. More precisely, for fixed $k \geq 2$ we define the

Received by the editor February 13, 1992.

1991 Mathematics Subject Classification. Primary 11 L03. 
sum

$$
S(m, x)=\sum_{n=0}^{m} \zeta^{b(n)} x^{n},
$$

where $m \geq 1, \zeta=\exp (2 \pi i / k)$, and $x$ is an arbitrary complex number. We also define $S(0, x)=1$ and $S(-1, x)=0$. For the more general sums the rotation property is replaced by the following:

Rotation Plus Translation Theorem. For $n \geq 0$ we have

$$
\begin{aligned}
S\left(n k^{2}+d_{1} k+d_{0}, x\right)= & x^{n k^{2}} \zeta^{b(n)} S\left(d_{1} k+d_{0}, x\right) \\
& +S(k-1, x) S\left(k-1, x^{k}\right) S\left(n-1, x^{k^{2}}\right) .
\end{aligned}
$$

The proof is based on the following lemma.

Lemma. If $m \geq 1$ and $0 \leq d_{0}<k$, we have

$$
S\left(k m+d_{0}, x\right)=S\left(m-1, x^{k}\right) S(k-1, x)+x^{m k} \zeta^{b(m)} S\left(d_{0}, x\right) .
$$

\section{Proofs}

Proof of the Lemma. In the sum defining $S\left(k m+d_{0}, x\right)$ write $n=\mu k+\nu$, and separate terms with $0 \leq \mu \leq m-1$ from those with $\mu=m$. Note also that $b(\nu)=\nu$ if $\nu<k$ and that $b(\mu k+\nu)=b(\mu)+\nu$ if $\nu<k$. Then we have

$$
\begin{aligned}
S\left(k m+d_{0}, x\right) & =\sum_{\mu=0}^{m-1} \sum_{\nu=0}^{k-1} \zeta^{b(\mu k+\nu)} x^{\mu k+\nu}+\sum_{\nu=0}^{d_{0}} \zeta^{b(m k+\nu)} x^{m k+\nu} \\
& =\sum_{\mu=0}^{m-1} \zeta^{b(\mu)} x^{\mu k} \sum_{\nu=0}^{k-1} \zeta^{\nu} x^{\nu}+x^{m k} \zeta^{b(m)} \sum_{\nu=0}^{d_{0}} \zeta^{\nu} x^{\nu} \\
& =S\left(m-1, x^{k}\right) S(k-1, x)+x^{m k} \zeta^{b(m)} S\left(d_{0}, x\right) .
\end{aligned}
$$

Note. If $m=0$, then $S\left(k m+d_{0}, x\right)=S\left(d_{0}, x\right)$, and this case is also covered by the lemma because of the convention $S(-1, x)=0$.

Proof of the Theorem. Write $n k^{2}+d_{1} k+d_{0}=m k+d_{0}$, where $m=n k+d_{1}$, and apply the lemma with $m=n k+d_{1}$ to obtain

$$
\begin{aligned}
S\left(n k^{2}+d_{1} k+d_{0}, x\right)= & S\left(n k+d_{1}-1, x^{k}\right) S(k-1, x) \\
& +x^{n k^{2}+d_{1} k} \zeta^{b\left(n k+d_{1}\right)} S\left(d_{0}, x\right) \\
= & S\left(n k+d_{1}-1, x^{k}\right) S(k-1, x) \\
& +x^{n k^{2}} \zeta^{b(n)} x^{d_{1} k} \zeta^{d_{1}} S\left(d_{0}, x\right) .
\end{aligned}
$$

Now apply the lemma to the sum $S\left(n k+d_{1}-1, x^{k}\right)$ to get

$$
S\left(n k+d_{1}-1, x^{k}\right)=S\left(n-1, x^{k^{2}}\right) S\left(k-1, x^{k}\right)+x^{n k^{2}} \zeta^{b(n)} S\left(d_{1}-1, x^{k}\right) \text {. }
$$

This gives us

$$
\begin{aligned}
S\left(n k^{2}+d_{1} k+d_{0}, x\right)= & S\left(n-1, x^{k^{2}}\right) S\left(k-1, x^{k}\right) S(k-1, x) \\
& +x^{n k^{2}} \zeta^{b(n)} S\left(d_{1}-1, x^{k}\right) S(k-1, x) \\
& +x^{n k^{2}} \zeta^{b(n)} x^{d_{1} k} \zeta^{d_{1}} S\left(d_{0}, x\right) .
\end{aligned}
$$


Applying the lemma once more with $m=d_{1}$, we find

$$
S\left(d_{1} k+d_{0}, x\right)=S\left(d_{1}-1, x^{k}\right) S(k-1, x)+x^{d_{1} k} \zeta^{b\left(d_{1}\right)} S\left(d_{0}, x\right) .
$$

Note that $b\left(d_{1}\right)=d_{1}$, so $\zeta^{d_{1}}=\zeta^{b\left(d_{1}\right)}$. Multiply the last displayed equation by $x^{n k^{2}} \zeta^{b(n)}$ and subtract from the previous equation to obtain the theorem.

\section{SPECIAL CASES AND APPLICATIONS}

(a) The factor $S(k-1, x)$ that appears in the main theorem is a geometric sum given by

$$
S(k-1, x)=\sum_{n=0}^{k-1} \zeta^{b(n)} x^{n}=\sum_{n=0}^{k-1}(\zeta x)^{n}= \begin{cases}k & \text { if } \zeta x=1 \\ \frac{1-x^{k}}{1-\zeta x} & \text { if } \zeta x \neq 1\end{cases}
$$

Similarly, we have

$$
S\left(k-1, x^{k}\right)= \begin{cases}k & \text { if } \zeta x^{k}=1, \\ \frac{1-x^{k^{2}}}{1-\zeta x^{k}} & \text { if } \zeta x^{k} \neq 1 .\end{cases}
$$

(b) When $x=\zeta^{j}$, the sum $S(m, x)$ reduces to the Lehmer sum $S_{j}(m)$. In this case $x^{k}=1$ and $\zeta x^{k}=\zeta \neq 1$, hence the geometric sum $S\left(k-1, x^{k}\right)$ vanishes and there is no translation term in the theorem.

(c) When $x=\zeta^{1 / k}$, then $x^{k}=\zeta, x^{k^{2}}=\zeta^{k}=1$, and $\zeta x^{k}=\zeta^{2} \neq 1$ if $k \geq 3$. In this case the geometric sum $S\left(k-1, x^{k}\right)$ vanishes and again there is no translation term in the theorem. In other words, we have the following pure rotation theorem for $k \geq 3$ :

$$
S\left(n k^{2}+d_{1} k+d_{0}, \zeta^{1 / k}\right)=\zeta^{b(n)} S\left(d_{1} k+d_{0}, \zeta^{1 / k}\right) .
$$

(d) Take $d_{0}=k-1$ in the lemma. This gives us

$$
\begin{aligned}
S(k m+k-1) & =S\left(m-1, x^{k}\right) S(k-1, x)+x^{m k} \zeta^{b(m)} S(k-1, x) \\
& =S(k-1, x)\left\{S\left(m-1, x^{k}\right)+x^{m k} \zeta^{b(m)}\right\} \\
& =S\left(k-1, x^{k}\right) S\left(m, x^{k}\right) .
\end{aligned}
$$

In particular, if $m=k-1$, we have $k m+k-1=k^{2}-1$, and we obtain

$$
S\left(k^{2}-1, x\right)=S(k-1, x) S\left(k-1, x^{k}\right) .
$$

(e) Take $d_{0}=k-1$ and $d_{1}=k-1$ in the main theorem to get

$$
\begin{aligned}
& S\left(n k^{2}+(k-1) k+k-1, x\right) \\
& \quad=x^{n k^{2}} \zeta^{b(n)} S\left(k^{2}-1, x\right)+S(k-1, x) S\left(k-1, x^{k}\right) S\left(n-1, x^{k^{2}}\right) .
\end{aligned}
$$

Because of (1) and the definition of $S\left(n, k^{2}\right)$ this becomes

$$
S\left(n k^{2}+(k-1) k+k-1, x\right)=S(k-1, x) S\left(k-1, x^{k}\right) S\left(n, x^{k^{2}}\right) .
$$

In particular, if $n=k-1$, we find

$$
S\left(k^{3}-1, x\right)=S(k-1, x) S\left(k-1, x^{k}\right) S\left(k-1, x^{k^{2}}\right) .
$$


(f) Relations (1) and (3) can be extended by induction to give us (4) $S\left(k^{\alpha}-1, x\right)=S(k-1, x) S\left(k-1, x^{k}\right) S\left(k-1, x^{k^{2}}\right) \cdots S\left(k-1, x^{k^{\alpha-1}}\right)$ for every integer $\alpha \geq 2$. In particular, if we choose $x$ so that $x=x^{k}$, then equation (4) becomes

$$
S\left(k^{\alpha}-1, x\right)=S(k-1, x)^{\alpha} .
$$

For $x \neq 0$ the relation $x=x^{k}$ is equivalent to $x^{k-1}=1$, and is satisfied, in particular, for

$$
x=\zeta_{j}=\exp (2 \pi i / j) \text {, }
$$

where $j \mid(k-1)$. In this case equation (5) becomes

$$
S\left(k^{\alpha}-1, \zeta_{j}\right)=S\left(k-1, \zeta_{j}\right)^{\alpha} \text {. }
$$

By (a), $S\left(k-1, \zeta_{j}\right)$ is a geometric sum given by

$$
S\left(k-1, \zeta_{j}\right)=\frac{1-\zeta_{j}}{1-\zeta_{k} \zeta_{j}}=\frac{1-e^{2 \pi i / j}}{1-e^{2 \pi i / j} e^{2 \pi i / k}}=e^{-\pi i / k} \frac{\sin \left(\frac{\pi}{j}\right)}{\sin \left(\frac{\pi}{j}+\frac{\pi}{k}\right)} .
$$

In a letter to the author [3], Emma Lehmer made the following observations: "If $j \mid(k-1)$ the numbers $S\left(k-1, \zeta_{j}\right)$ have absolute value $<1$ for $3 \leq j<k$, and your equation $(6)$ shows that

$$
S\left(k^{\alpha}-1, \zeta_{j}\right) \rightarrow 0 \text { as } \alpha \rightarrow \infty \text { for } 3 \leq j<k .
$$

This means that the graph of the corresponding sum returns to the neighborhood of 0 infinitely often, but is no longer periodic, nor is it 'picturesque,' as you can see by the enclosed diagram for $k=4$ and $j=3$."

(g) When $|x|<1$ the infinite series $S(x)=\lim _{m \rightarrow \infty} S(m, x)$ converges and is given by

$$
S(x)=\sum_{n=0}^{\infty} \zeta^{b(n)} x^{n}
$$

If we let $n \rightarrow \infty$ in our main theorem, the first term tends to zero because $|x|<1$, and we find

$$
S(x)=S(k-1, x) S\left(k-1, x^{k}\right) S\left(x^{k^{2}}\right)=\frac{1-x^{k}}{1-\zeta x} \frac{1-x^{k^{2}}}{1-\zeta x^{k}} S\left(x^{k^{2}}\right) .
$$

Repeated use of this functional equation gives the infinite product representation

$$
S(x)=\prod_{\nu=0}^{\infty}\left(1-x^{k^{\nu+1}}\right) /\left(1-\zeta x^{k^{\nu}}\right)
$$

in agreement with equation (1) in the Lehmer paper [1].

\section{BIBLIOGRAPHY}

1. D. H. Lehmer and Emma Lehmer, Picturesque exponential sums. I, Amer. Math. Monthly 86 (1979), 725-733.

2. D. H. and Emma Lehmer, Picturesque exponential sums. II, J. Reine Angew. Math. 318 (1980), 1-19.

3. Emma Lehmer, Personal communication to Tom M. Apostol, dated January 4, 1980.

Department of Mathematics, 253-37, California Institute of Technology, Pasadena, CALIFORNIA 91125

E-mail address: apostol@caltech.bitnet 